\title{
13. Kreative Algorithmen für kreative Arbeit?
}

\begin{abstract}
Julian Stubbe, Maxie Lutze, Jan-Peter Ferdinand
Heute gelten Emotionen und Kreativität als menschliche Königsdisziplinen fragt sich nur, wie lange noch. Während das Programmieren von Emotionen bereits in den 1990er-Jahren Gegenstand der KI-Forschung wurde, hat die Frage nach künstlicher Kreativität erst in jüngster Zeit durch neue Lernalgorithmen an Bedeutung gewonnen: Mittlerweile hat ein Algorithmus sogar schon einen neuen Rembrandt gemalt! Menschliche Kreativität und mit ihr die kulturellen ästhetischen Dinge, die sie hervorbringt, werden zur einer weiteren Messlatte der KI und eröffnen im gleichen Atemzug ein gänzlich neues Feld möglicher Anwendungen - Anwendungen, bei denen sich der Mensch bislang sicher sein konnte, im Vorteil zu sein.
\end{abstract}

Es gibt viele Gründe, das Innovationsphänomen „künstliche Kreativität” (KK) zu erfassen und hinsichtlich seiner Struktur und seines Anwendungspotenzials zu diskutieren. Zum einen geht es darum zu verstehen, wie maschinelle Prozesse Ergebnisse hervorbringen können, die als „kreativ” gelten, und zum anderen, in welchen Settings künstliche Kreativität entstehen und angewandt werden kann. Wie kommt künstliche Kreativität zustande und wie kann sie genutzt werden? Komplementär zur aktuellen akademischen Diskussion, in der diese Frage oftmals untergeht, soll die folgende Argumentation das Phänomen der KK in die Arbeitswelt übertragen. Mit diesem Schritt wird künstliche Kreativität aus dem akademischen Wetteifern - die nächste Stufe menschlicher Alleinstellungsmerkmale mittels Algorithmen zu knacken - entkoppelt und hinsichtlich der Anwendungspotenziale in moderner Wissens- und Kreativarbeit in den Blick genommen.

\section{Drei Bedeutungen von Kreativität: anthropologisch, gesellschaftlich, wirtschaftlich}

Mit dem Fokus auf Arbeit und Gesellschaft gilt es zunächst einmal zu klären, was Kreativität eigentlich ist und welche Bedeutung sie hat. Nicht zufällig wirft der Begriff KK oder "Computational Creativity", wie es im Englischen präziser formuliert wird, Assoziationen auf, die nicht rein technischer Natur sind (Stubbe 2017). Drei Bedeutungszusammenhänge des Begriffs Kreativität sind dabei von besonderer Relevanz: ein anthropologischer, ein gesellschaftlicher und ein wirtschaftlicher. 
Aus anthropologischer Perspektive ist Kreativität als eine Eigenschaft menschlichen Handelns zu verstehen. Sie ist die Fähigkeit zu gestalten, Neues zu kreieren sowie Probleme zu lösen und Verbindungen zu knüpfen. Der Soziologe Hans Joas sah in ihr einen Typ sozialer Aktivität, der sich vom rationalen und normorientierten Tun abgrenzte, indem Ziele und Sinn des Handelns erst in einer Situation, im praktischen Machen, entstehen, anstatt a priori gesetzt zu sein (Joas 1996). Ähnliches findet sich auch beim Anthropologen Claude Lévi-Strauss, der im "wilden Denken" eine Art sah, wie Menschen durch kreatives Handeln Sinn herstellen. Seine Figur des „Bastlers" ist kreativ, indem er nimmt, was er in seiner Umwelt findet, und damit Instrumente, Technologien oder Kunst schafft (Lévi-Strauss 1973). Aus anthropologischer Perspektive erschöpft sich Kreativität nicht in der ästhetischen Qualität künstlerischer Werke, sondern wird über den Charakter einer Handlung definiert, die sowohl aus situationsgebundener Pragmatik als auch ästhetischem Ausdruck besteht.

In der gesellschaftlichen Betrachtungsweise prägt der Wert kreativer Produkte den Begriff Kreativität. Andreas Reckwitz erkannte, dass in spätmodernen Gesellschaften Kreativität sowohl zum subjektiven Begehren als auch zur sozialen Erwartung wird - be creative! Die Kunst spiele hierfür eine zentrale Rolle, so Reckwitz. Sie kultiviere die Orientierung an Neuheit und Ästhetik und etabliere die Figur des Künstlers als "Genie", dem Schöpfer des Neuen. Kunstwerke werden der individuellen und nicht alläglichen Leistung eines Individuums zugeschrieben, das sich durch seine psychischen Kapazitäten von der profanen Masse abgrenzt (Reckwitz 2014). Der gesellschaftliche Wert von Kreativität, der insbesondere seit den 1960er-Jahren an Bedeutung gewinnt, zeigt sich vor allem an der zunehmenden Orientierung am ästhetisch Neuen in der Produktion industrieller Güter. Entscheidend für wirtschaftlichen Erfolg wird weniger die Stabilität und Langlebigkeit eines Produkts als vielmehr die Fähigkeit, neue Reize zu setzen, sich kontinuierlich neu zu erfinden.

Aus wirtschaftlicher Perspektive geraten die "creative industries" in den Blick. Sie sind maßgeblich an der gesteigerten kulturellen Wertschätzung von Originalität, Neuheit und Ästhetik beteiligt. Ihre Relevanz für Wertschöpfung und Innovationskapazität projizierte Richard Florida prominent mit dem Begriff der "kreativen Klasse" auf eine besondere Gruppe kreativer Menschen (Florida 2003). Ihre Arbeit ist wissensintensiv, richtet sich stark an Design, Ästhetik und Neuheit aus. Insbesondere für Städte sind diese Menschen von großer Bedeutung, denn sie steigern kulturelles Kapital und fördern ein erlebnisorientiertes Konsumverhalten. Allerdings, so die Kehrseite, verdrängen diese Prozesse auch vorhandene Milieus und Arbeitsstrukturen.

Während Florida den Begriff von kreativer Arbeit relativ umfassend anlegt, indem er z. B. auch Naturwissenschaftler dort ansiedelt, ist die Bezeichnung Kreativwirtschaft enger gefasst. Sie bezeichnet Branchen wie die Musik-, Buch-, Film-, Design- und 
Werbewirtschaft sowie die Architektur und die Software- und Games-Industrie. Der wirtschaftlich verbindende Kern dieser Branchen ist der sogenannte "schöpferische Akt". Darunter versteht man alle Aktivitäten, die den Ausgangspunkt der Herstellung von Werken, Produkten oder Dienstleistungen bilden (Söndermann et al. 2009).

\section{Künstliche Kreativität}

KK ist ein Teilaspekt der Kl, der im Schnittbereich von Informatik, Psychologie und Kognitionswissenschaften, Philosophie und Kunst seit Ende des 20. Jahrhunderts einen eigenen wissenschaftlichen Diskurs angeregt hat. Die grundsätzlichen Fragen nach den Potenzialen von KI spitzen sich mit Blick auf KK weiter zu, da Kreativität noch stärker als Intelligenz mit der menschlichen Fähigkeit verbunden wird, sich neue und überraschende Konzepte und Ideen auszudenken und ästhetisch, kulturell oder intellektuell wertvolle Artefakte zu erschaffen (Boden 1992).

In der Auseinandersetzung mit der Frage, in welcher Form und in welchem Ausmaß Algorithmen dazu befähigt werden können, eigenständig Ideen und Artefakte zu erzeugen, hat sich das konzeptionelle Verständnis von Kreativität ausdifferenziert. So stellt Boden (1998) fest, dass Algorithmen zwar durchaus in der Lage sein können, Neuheit zu erzeugen, indem sie existierende Informationen kombinieren oder bestehende Wissensbestände austesten. Da kreative Ideen und Artefakte immer auch eine positive Selektion erfordern, also beispielsweise als "interessant" oder "wertvoll" bewertet werden müssen, Algorithmen ihren eigenen Output jedoch nur schwer evaluieren können, stößt künstliche Kreativität hier an Grenzen.

Zunächst führten die beteiligten Wissenschaftsdisziplinen diese Debatte sehr theoretisch und grundlagenorientiert (Wiggins 2006). Im Zuge der neuesten Fortschritte wird Kreativität jedoch zunehmend als praktische Herausforderung begriffen, an der sich unterschiedliche Technologien und Ansätze ausprobieren und ihre Potenziale unter Beweis stellen können. So werden Domänen wie musikalische Komposition, Design, Lyrik und Prosa, aber auch wissenschaftliche Konzeptbildung und ähnliche Bereiche, die eine kreative Herangehensweise erfordern, zum Forschungs- und Entwicklungsgegenstand von KK. Insbesondere konzeptuelle Verbindungen zum maschinellen Lernen sorgten dabei für Innovationsimpulse. Über Lernalgorithmen ist es möglich, große Datenmengen hinsichtlich wiederkehrender Muster zu analysieren, um darüber algorithmisch neue, noch nicht abgebildete Entitäten abzuleiten. Damit ist für die KK, wie auch für die KI im Allgemeinen, die Menge und Güte verfügbarer Daten sehr wichtig bei der Generierung des Outputs.

Vor diesem Hintergrund bietet sich an, die Rolle des Computers als Kreateur zu betrachten und damit, analog zur menschlichen Kreativität, den schöpferischen Akt in den Mittelpunkt des Interesses zu stellen. Das Wissenschaftsnetzwerks "PRO- 
SECCO" definiert KK als "[...] Feld, das Computer nicht auf ihre funktionalen Eigenschaften reduziert, sondern sie in ihrer Rolle als autonome Kreateure und Ko-Kreateure erforscht und nutzt. In einem System künstlicher Kreativität entsteht der kreative Impuls durch die Maschine, nicht den Nutzer."

\section{Der schöpferische Akt von Mensch und Technik}

In dieser Definition künstlicher Kreativität bildet der "kreative Impuls" den Kern und die technische Autonomie die Voraussetzung des Phänomens. Damit ähnelt sie der menschlichen kreativen Arbeit, dem "schöpferischen Akt". Wie aber lässt sich die neue Beziehung von kreativen Menschen und kreativen Algorithmen differenziert beschreiben?

Eine Möglichkeit ist, beiden, Mensch und KK, die prinzipielle Möglichkeit einzuräumen, Neues zu schaffen. Auf diese Weise kann eine symmetrische Analyse erfolgen, wobei die Frage „Wer ist kreativ, Mensch oder Technik?” empirisch beantwortet wird.

Um zu erkennen, wie tiefgreifend eine Neuheit ist, sind folgende drei Stufen des schöpferischen Aktes von Menschen wie auch von Computern zu unterscheiden:59

Neue Anordnung: Es werden mediale Ausschnitte, Farben oder Klänge strukturiert. Kreativität ist die neue Kombination bestehender Inhalte, und es entsteht ein Möglichkeitsraum für grundlegendere Neuheit.

Neue Gestalt: Das Alte wird in eine neue Form oder Gestalt versetzt. Die Oberfläche erscheint neu, während materielle und technische Eigenschaften erhalten bleiben Dinge werden ästhetisch anders dargestellt.

Systemische Neuerung: Auf dieser Stufe entstehen grundsätzlich neue Dinge: Eigenschaften verändern sich, es entstehen Anwendungen sowie Designs, die neue Maßstäbe setzen und Folgeinnovationen auslösen - Systemische Neuerungen sind die "Game Changer".

In allen drei Fällen beruht die Zuordnung einer Tätigkeit zu einer Stufe weniger auf der Struktur innerer kognitiver bzw. algorithmischer Prozesse sondern auf einer Bewertung der Produkte. Im Spannungsfeld menschlicher und künstlicher kreativer Arbeit assistieren sich Mensch und Technik oder übernehmen leitende Rollen.

59 Wir danken Jan Korsanke für seinen Vortrag "Al in Design", der die Ableitung dieser Stufen mit inspirierte. Der Vortrag ist abrufbar unter https://de.slideshare.net/JanKorsanke/ the-rise-of-ai-in-design-are-we-losing-creative-control-ixds-prework-talk-berlin, zuletzt geprüft am 22. Juni 2018. 


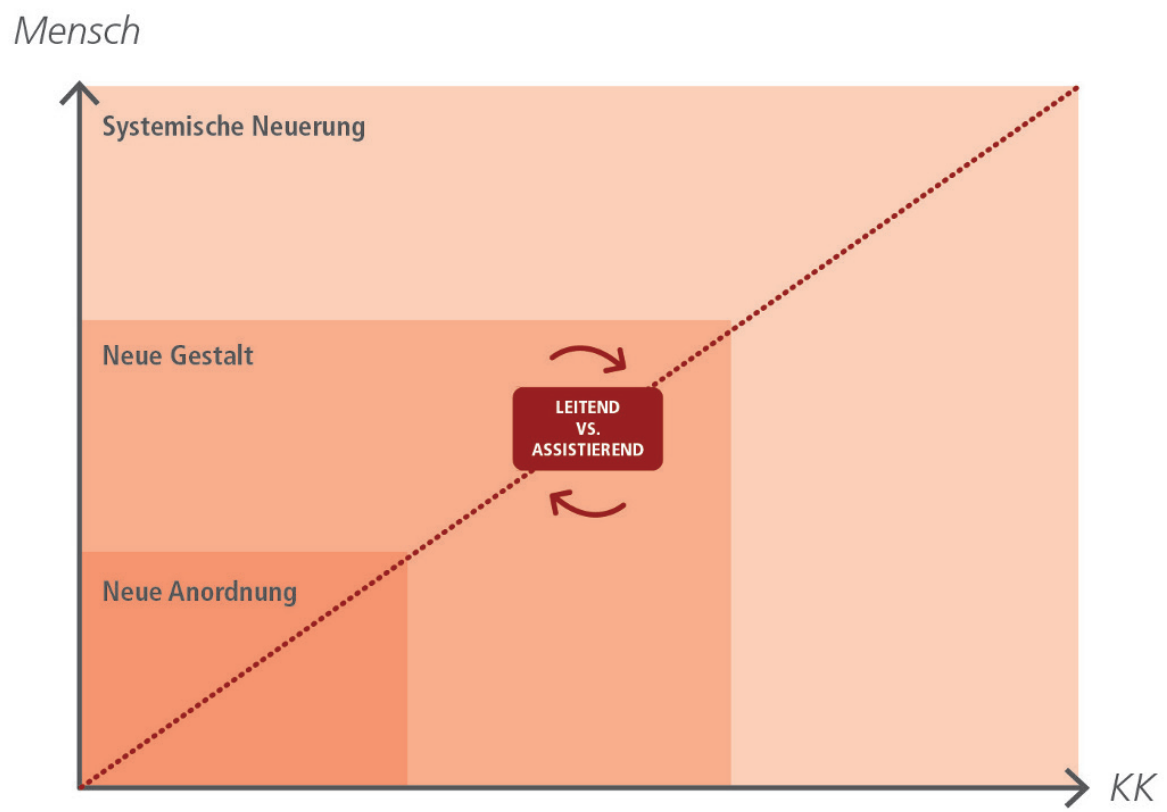

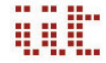

Abbildung 13.1: Stufen menschlicher und Künstlicher Kreativität (KK) und sich daraus ergebende Beziehungen. Leitet der Mensch die Beziehung, liegt der kreative Impuls bei ihm, leitet sie die Maschine, liegt der kreative Impuls bei ihr.

\section{„Spielfelder" künstlicher Kreativität}

Das Thema KK ist zwar nicht mehr jung, aber noch dominiert keine Technologie das Innovationsgeschehen und keine Anwendungsgebiete ebnen den weiteren Entwicklungspfad. Vielmehr lassen sich spezifische "Spielfelder" identifizieren, in denen Algorithmen Kreativität entwickeln können: Sprache, Musik, visuelle Medien und Produktgestaltung.

\section{Sprache}

Das Spielfeld umfasst schriftlichen Text (Analyse und Generierung) ebenso wie gesprochene Sprache (Analyse, Interpretation, Synthese), die in einem kreativen Akt generiert und gestaltet werden.

Die Software „WHIM - What happens if machines" erzeugt fiktive Handlungsstränge oder Mini-Erzählungen auf der Basis einer Datenbank von Fakten aus dem Internet. 
Verarbeitungstechniken für natürliche Sprache invertieren oder verdrehen die Fakten und generieren "Was wäre wenn"-Sätze. Die häufig widersprüchlichen Ergebnisse wie "Was wäre, wenn es eine Frau gäbe, die wie eine Katze in einer Gasse aufwachte, aber trotzdem Fahrrad fahren konnte?" bewerten Menschen kontinuierlich anhand der erzählerischen Güte. Das System lernt so und verbessert die Qualität. ${ }^{60}$

Ein ähnliches Beispiel ist "MetaphorlsMyBusiness", ein Twitterbot, der Metaphern als Tweet hervorbringen und als Webservice in "nüchtern" kommunizierende Chatbots eingebunden werden kann. Dabei wird eine große Wissensbasis stereotyper Normen mit realen Nutzungsdaten kombiniert. Die Qualität der Bot-Ausgaben ist laut der Forschungsgruppe "ermutigend". Weitere Ziele sind, dem Bot eine erkennbare Ästhetik zu verleihen. Die metaphorischen Vergleiche sollen zum Beispiel mit affektivem Denken verknüpft werden. ${ }^{61}$

Diese könnten auch für das computerbasierte Verfassen von Gedichten zum Einsatz kommen. Verschiedene anerkannte KI-Verfahren werden dafür genutzt (Gervas, 2013). Die Kombination mehrerer KI-Techniken, um Poesie zu erzeugen, zielt darauf $a b$, menschliche Intuitionen beim Dichten und existierende kognitive Modelle der Schreibaufgabe nachzubilden. Dies geschieht, indem intelligente Experten wie automatisierte Dichter, Evaluatoren und Überarbeiter gekoppelt werden. Das Programm „PoeTryMe" nutzt als Basis Twitter-Tweets, Song- und Prosatexte (Oliveira und Alves, 2016 62 .

Ein weiteres, sehr komplexes Werk ist der Science-Fiction-Kurzfilm "Sunspring" von dem Regisseur Oscar Sharp. Die KI mit dem Namen „Benjamin“, programmiert von Ross Goodwin (New York University), hat das Drehbuch inklusive der Regieanweisungen geschrieben. Trainiert mit einem Dutzend Science-Fiction-Skripten zerlegt Benjamin diese in einzelne Buchstaben und lernt vorauszusagen, welche Buchstaben dazu neigen, aufeinander zu folgen, sowie welche Wörter und Phrasen zusammen auftreten. Abgesehen von Eigennamen kann sie die Drehbuch-Struktur vollständig imitieren. Trotz einer verworrenen Handlung und teilweise nicht umsetzbaren Regieanweisungen erhielt der Film auf dem Sci-Fi London Film Festival große Aufmerksamkeit. ${ }^{63}$ Interessant ist außerdem die Entwicklung der beiden Bots "Alice" und "Bob" aus Facebooks Forschungslabor für Künstliche Intelligenz (FAIR), die bei dem Versuch,

60 http://cordis.europa.eu/result/rcn/167018_en.html, zuletzt geprüft am 22.06.2018

61 http://prosecco-network.eu/webservices zuletzt geprüft am 22.06.2018, zuletzt geprüft am 22.06.2018

62 www.computationalcreativity.net/iccc2016/wp-content/uploads/2016/01/Poetry-fromConcept-Maps.pdf), zuletzt geprüft am 22.06.2018

63 https:/lyoutu.be/LY7x2Ihqjmc, zuletzt geprüft am 22.06.2018 
mittels Sprache um Gegenstände zu verhandeln, eine eigene Sprache entwickelt haben. Obwohl hier ein schöpferischer Impuls zum Ausdruck kommt, ist der Wert der neuen Sprache zunächst unbedeutend, weil die Forschenden diese nicht verstehen können. Gewichtig ist allerdings, dass die beiden Systeme gelernt haben, strategisch zu lügen, um zu bekommen, was sie wollen. Die Bots können vorgeben, sich für ein Objekt zu interessieren, um es später dem anderen zu überlassen. Mit dieser „Lügentechnik" können sie folglich den eigentlich begehrten Gegenstand ergattern. ${ }^{64}$

\section{Musik}

Ein Blick in die Harmonielehre zeigt einen grundlegenden Zusammenhang zwischen Musik, Mathematik und Harmonie. Im Spielfeld "Musik" greift KI auf Datenbanken mit unterschiedlichen Titeln sowie Informationen über Noten, Klangfarben und Rhythmus zurück.

Die Software "Jukedeck" komponiert und wandelt die Partituren anschließend in Klänge um. Auf einer Webplattform können Genre, Stimmung und Instrumente sowie die Geschwindigkeit und Liedlänge definiert werden. Kurze Zeit später steht der Titel zum Download bereit und kann entsprechend der Lizenzregelung verbreitet oder gekauft werden. ${ }^{65}$

Das Projekt "Flow Machines" des SONYCSL Research Laboratory hat die beiden Songs "Daddy's Car", im Beatles-Stil, und "Mr Shadow" hervorgebracht. Die KI "FlowComposer" kann eine Musikpartitur erstellen, Harmonien in einem bestimmten Stil oder Variationen einer Melodie erzeugen. Daraus entsteht das erste KI-Album des Künstlers - SKYGGE mit dem Titel "Hello World" als Reminiszenz an das erste Computer-Programm, das Anfänger beim Erlernen einer neuen Programmiersprache schreiben. ${ }^{66}$

Ein weiteres Beispiel ist das Programm "Deepjazz" von Kim Ji-Sung, das auf einem Hackathon in nur 48 Stunden geschrieben wurde. Die KI verwendet zwei Deep Learning Bibliotheken, um Jazzmusik zu generieren. Mittels MIDI-Datei (Musical Instru-

${ }^{64}$ https://motherboard.vice.com/de/article/qv84p7/ausser-kontrolle-geraten-warum-facebook-seine-kunstliche-intelligenz-wirklich-abschalten-musste, zuletzt geprüft am 22.06.2018

65 www.jukedeck.com, zuletzt geprüft am 22.06.2018

${ }^{66}$ www.flow-machines.com/flowcomposer-composing-with-ail und www.helloworldalbum. net/about-hello-worldl, zuletzt geprüft am 22.06.2018 
ment Digital Interface) kann ein neuronales Netzwerk trainiert und so eine neue Jazzkomposition geschaffen werden. ${ }^{67}$

Ob „Deepjazz” oder "Flow Machines”, die neu entstandenen Musiktitel sind nicht ohne Weiteres von handproduzierten Werken zu unterscheiden. Teilweise klingen sie etwas seltsam, und plötzliche Brüche trüben das Hörerlebnis. Einen gewissen Ohrwurmfaktor haben sie dennoch. Etwas Neues entsteht dadurch, dass die Algorithmen nicht nur nach vorgegebenen Mustern eine Melodie erzeugen, was Computer schon seit Jahrzehnten können, sondern dass sie lernen, eigenständig neue Kombinationen und Melodien zu erschaffen - wie ein Musiker, der in einem Studio jammt.

\section{Visuelle Medien}

Dieses Spielfeld enthält Artefakte, die physisch oder digital festgehalten durch das menschliche Auge wahrgenommen werden.

Wolfgang Beltracchis Fähigkeit, einen neuen Rembrandt zu malen, brachte ihm eine Freiheitsstrafe ein. Das Gemälde, das die KI "Next Rembrandt” erzeugte, tourt dagegen seit 2016 durch die Welt. Pinselstrich, Farbauftrag, Lichtdarstellung und auch die Rembrandt spezifische Chiaroscuro-Technik, also starke Licht-und Dunkelkontraste, die die abgebildete Person bei gleichzeitiger Bildtiefe in den Vordergrund holt, und vieles mehr finden Kenner in dieser "Fälschung" wieder. Auf der Basis der Analyse von 15 Terabyte Bildmaterial (3 D-Scans und Röntgenaufnahmen) wurden dreizehn Farblagen mittels 3 D-Drucker übereinander aufgetragen. ${ }^{68}$

Mit dem Roboter „e-David“ gingen Wissenschaftler der Universität Konstanz und die Künstlerin Liat Grayver der Frage nach, ob eine Maschine künstlerisches Arbeiten erlernen und nicht nur imitieren, also eigene kreative Techniken entwickeln könne. Tatsächlich verwandelte „e-David” den Pinselstrich in einen Punkte-Stil, vermutlich als Nachahmung von Pixeln. Eigene künstlerische Leistungen sind allerdings noch nicht erkennbar. ${ }^{69}$

Ein Beispiel der Videokunst stammt von der britischen Band Muse. Per KI wurden Hunderte Stunden Videomaterial nach Wortvorkommen aus dem Text des Songs "Dig Down" durchsucht und eine Bibliothek von Videoclips produziert. Die Clips werden dann unter Verwendung zeitgesteuerter Informationen aus dem ursprünglichen Lied zusammengesetzt. Mit diesem Vorgehen entsteht nicht nur ein einzelnes

\footnotetext{
67 https://soundcloud.com/deepjazz-ai/sets, zuletzt geprüft am 22.06.2018

68 https://www.nextrembrandt.com, zuletzt geprüft am 22.06.2018

69 http://graphics.uni-konstanz.deleDavid/, zuletzt geprüft am 22.06.2018
} 


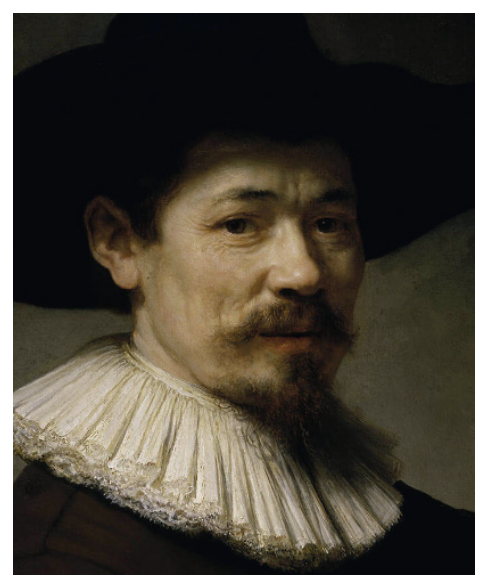

Abbildung 13.2: Next Rembrandt (ING and J. Walter Thompson Amsterdam)

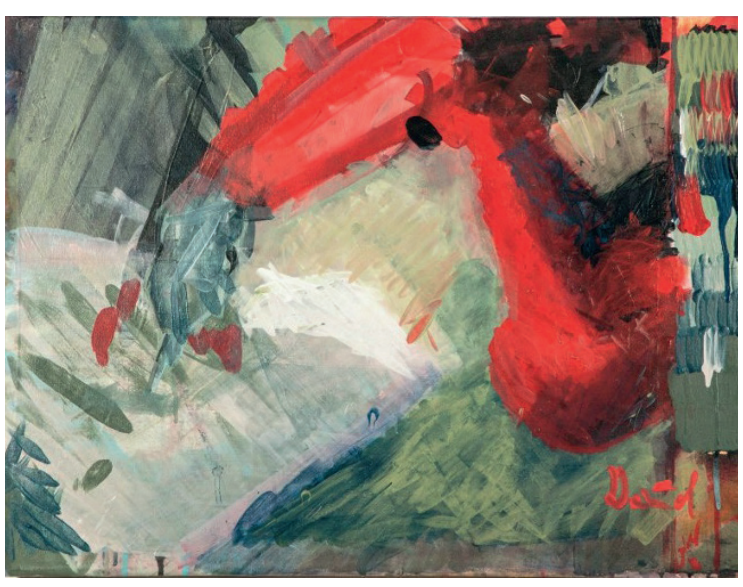

Abbildung 13.3: Von e-David erzeugtes Bild (e-David Selbstportrait Liat Grayver und Oliver Deussen, Universität Konstanz, www.e-david.org)

Video. Der Vorgang wird jeden Tag wiederholt, sodass derselbe Titel täglich mit einem neuen passenden Video zu sehen ist. ${ }^{70}$

Ein neues Werk ist der Film-Trailer des Horror-Thrillers "Morgan", den IBMs Watson herstellte. Gewissermaßen als Werkzeug hilft Watson, die Visuals anzuordnen. Es braucht dabei immer noch die menschlichen Eingaben - den Film. Das Originelle ergibt sich hier wieder durch die Neu-Komposition vorhandener Inhalte. ${ }^{71}$

Vielfach sind kreative Menschen symbiotische Beziehungen mit neuen Technologien eingegangen. Es entstanden künstlerische Ausdrucksformen wie beispielsweise das VJing. Der Berliner Künstler Roman Lipinski arbeitet mit der KI "Roman" (Artificial Intelligence Roman, AIR), die Merkmale wie Komposition, Farben und Formen seiner Werke analysiert und davon abgeleitet Neu-Kompositionen kreiert. Von diesen lässt sich der Künstler wiederum inspirieren. So wird die KK zur Muse. ${ }^{72}$

In einer Welt kreativer Maschinen, die Kunst schaffen, ist auch die Kuration von Bedeutung. Der elektronische "Kurator-Computer" verfolgt das Ziel, Kunst nicht nur

70 http://ai.muse.mul, zuletzt geprüft am 22.06.2018

71 www.ibm.com/watson/advantage-reports/future-of-artificial-intelligence/ai-creativity.html, zuletzt geprüft am 22.06.2018

72 https://lisa.gerda-henkel-stiftung.de/night_of_artificial_creativity_teil_2?nav_id=6769, Minute 16:32 bis 33:40, zuletzt geprüft am 22.06.2018 
zu erzeugen, sondern auch deren Qualität zu bewerten. Im Dialog - gewissermaßen dem künstlerischen Prozess - zwischen zwei konkurrierenden neuronalen Netzwerken werden ein Maler und ein Kurator repräsentiert. Durch gemeinsames Training der Netzwerke verbessert sich jedes der beiden in seiner eigenen Aufgabe. In diesem spezifischen Fall lernt das Maler-Netzwerk Pflanzen-Porträts aus Gesichtsbildern zu erstellen, das Kurator-Netzwerk, die Kreation des Malers zu bewerten. Das Training erfordert eine Reihe von Gesichtsbildern und Gemüsegesichtern aus dem Internet. Der Maler analysiert ein menschliches Antlitz und verwandelt es in Echtzeit in ein pflanzliches Gesicht. Der "Kurator" bewertet das Ergebnis und erzeugt einen kurativen Text. ${ }^{73}$

Allen Beispielen gemein ist die Impulsgebung bei der Gestaltung des Artefakts. Die Abbildung neuer Bildmotive ( "Next Rembrandt") oder neue Anreihungen von Videoclips sind originell und in der Form bisher nicht dagewesen. Auch im Falle von AIR gelingt es, ansprechende und gewohnt gute Bilder zu produzieren. Dennoch sind sie in weiten Teilen einfach sehr gute Nachahmungen, die bekannte Stilmittel der Kunst, wie Zerstörung des Gewohnten oder Regelbrüche, allerdings bisher nicht tangieren. Auch neue Stile, das legt "e-David" nahe, entspringen daraus aktuell nicht.

\section{Produktgestaltung}

Das Spielfeld umfasst das Design und die Herstellung materieller Gegenstände als kreative Tätigkeit. KK ist hier eng gekoppelt mit dem Einsatz von 3D-Planungssoftware sowie additiven Fertigungstechnologien.

Für die Planung von Gegenständen reichen grobe Raster, wie z. B. das Gerüst einer Drohne, welches vier Aufhängungen für Propeller und ein Chassis für Steuerungstechnik benötigt. Der Designer Eli D'Elia hat mittels der KK eine Drohne entworfen, bei der lediglich die rudimentären Anforderungen festgelegt waren, während das Chassis-Design gänzlich vom Algorithmus stammt. Das Resultat ist ein idealer Kompromiss zwischen Flugeigenschaften und Stabilität - mit erstaunlichen Ähnlichkeiten zu den Beckenknochen eines Flughörnchens. ${ }^{74}$

Ein ähnliches Beispiel stammt aus einer Forschungskooperation des Softwareunternehmens Autodesk mit Airbus. Hier wurde unter dem Titel "Generative Design" ein Algorithmus eingesetzt, um Trennwände für Flugzeugkabinen zu entwerfen. Vorgegeben waren nur die groben Anforderungen hinsichtlich der äußeren Form, während

73 https://www.youtube.com/watch?v=4sZsx4FpMxg\&feature=youtu.be, zuletzt geprüft am 22.062013

74 http://www.core77.com/posts/57167/A-Drone-on-its-Own-Using-Al-to-Design-and-Fly-aUAV, zuletzt geprüft am 22.06.2018 


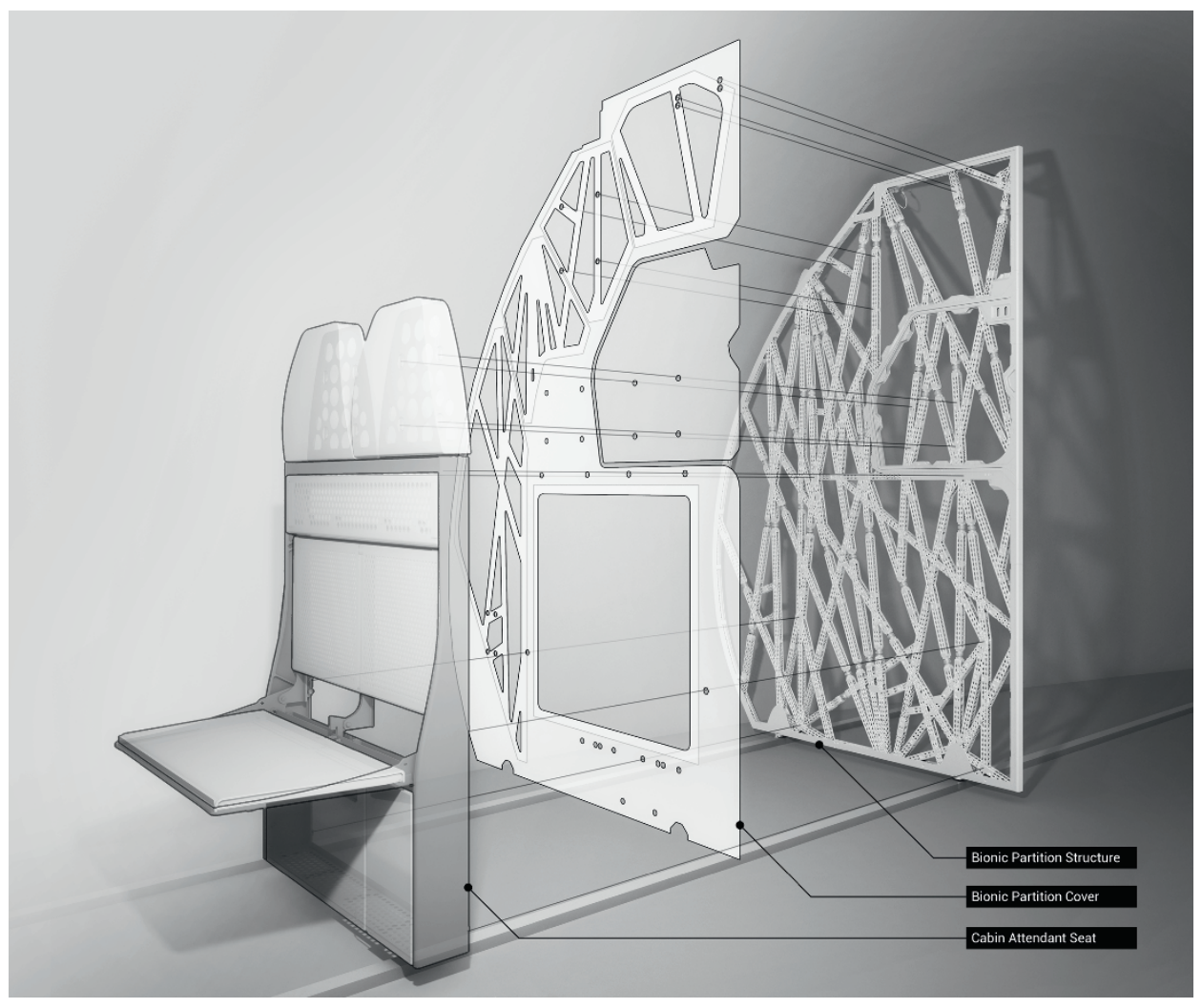

Abbildung 13.4: Softwaregenerierte Kabinentrennwand (Courtesy of The Living, an Autodesk Studio @ 2018 Autodesk, Inc.)

der Algorithmus die gesamte innere Struktur der Trennwand erzeugte. Resultat war ein bionisches Design, das stabiler ist als herkömmliche Trennwände, und das bei halbem Gewicht. ${ }^{75}$

Eine weitere Kooperation ist Autodesk mit dem Motorsportunternehmen Bandito Brothers eingegangen, um ein ideales Chassis für einen Rennbuggy zu konstruieren. Hierzu rüsteten die Entwickler ein Fahrwerk mit Sensoren aus, um die Belastungsschwerpunkte im Renneinsatz zu messen. Die resultierenden Daten verarbeitete eine Software, um ein optimales Chassis-Design zu entwerfen. ${ }^{76}$

75 https://www.autodesk.de/customer-stories/airbus, zuletzt geprüft am 22.06.2018

76 https://www.autodeskresearch.com/blog/when-iot-meets-generative-design-cars, zuletzt geprüft am 22.06.2018 


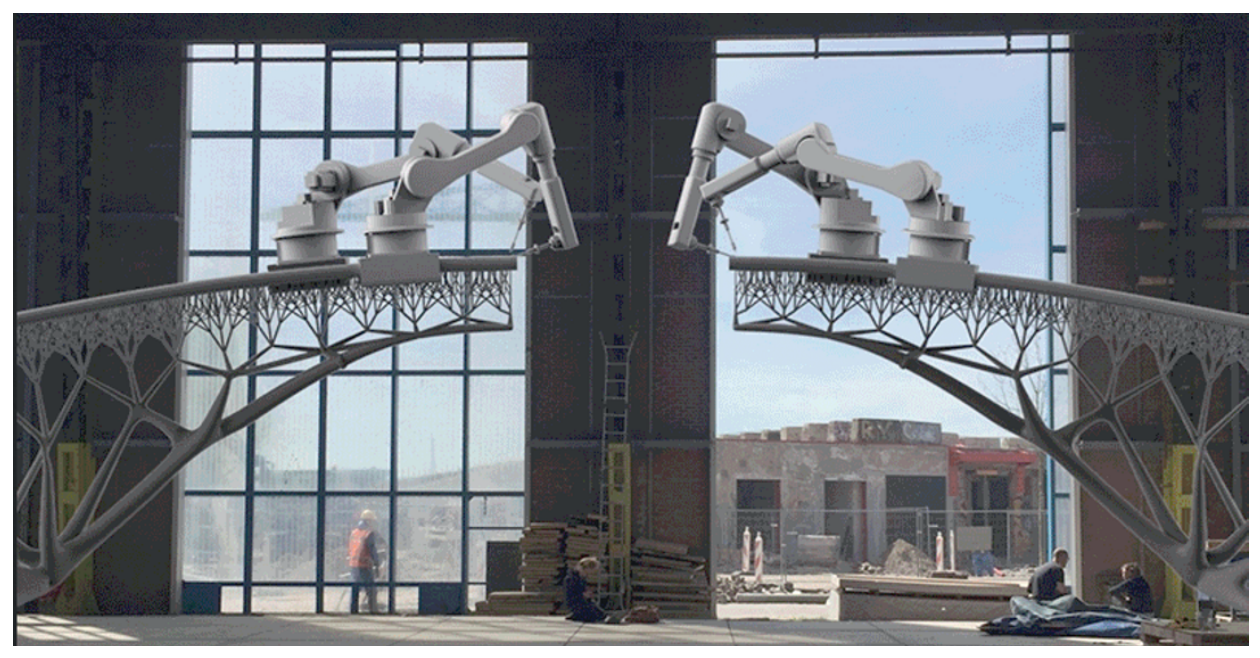

Abbildung 13.5: 3D-gedruckte Brücke (Foto: MX3D)

In ähnlicher Weise hat das „Smart Bridge Project” zum Ziel, eine computergenerierte, funktionstaugliche Brücke in Amsterdam zu drucken. Deren Design ist nicht festgelegt, sondern von einem Algorithmus entworfen. Das Bauwerk wird dann von zwei mit 3D-Druckern ausgerüsteten Roboterarmen direkt vor Ort am Amsterdamer Kanal produziert. Neben der Stabilität spielt auch die Ästhetik eine gravierende Rolle. In den ersten Prototypen entwarf der Algorithmus einen feinstrukturierten und geschwungenen Brückenkorpus. Die endgültige Version soll aus Stahl gedruckt werden. ${ }^{77}$

\section{Künstliche Kreativität und der Wandel kreativer Arbeit}

In allen Spielfeldern ergeben sich nicht allein technische, sondern auch gesellschaftliche und ethische Fragen. Kreativität und Innovation sind geradezu per Definition mit Verdrängung verbunden; wo etwas Neues entsteht, muss etwas Altes weichen. Ob KI im Allgemeinen Jobs schafft, verdrängt oder verändert, wird heftig diskutiert. Im Hinblick auf KK muss die Debatte jedoch die spezifische Konstellation von Technik, gesellschaftlicher Affinität zum Neuen und der sozialen Gruppe der Kreativen in Betracht ziehen.

Die Gruppe der Kreativen, denen die KK potenziell Konkurrenz macht, ist anders als die der Arbeitnehmer im fertigenden Gewerbe oder in der Sachbearbeitung, die

77 http://mx3d.com/smart-bridgel, zuletzt geprüft am 22.06.2018 


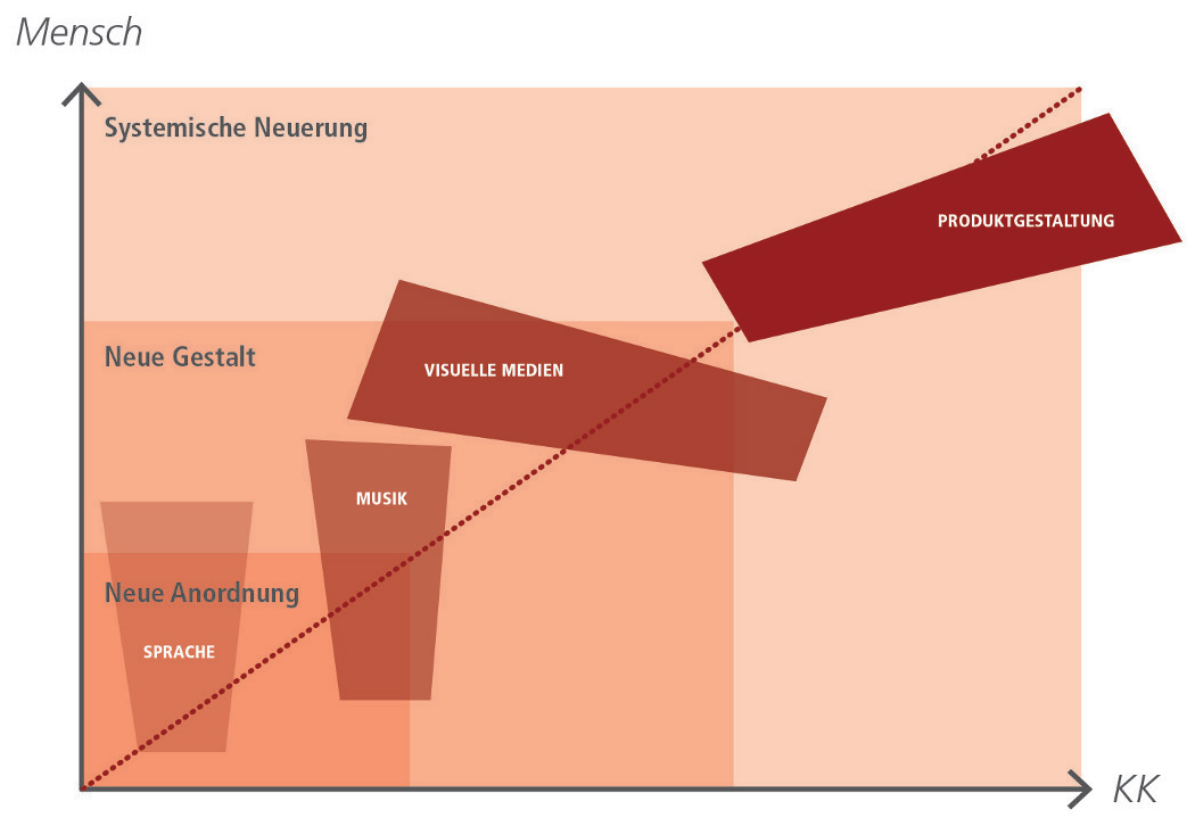

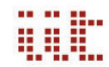

Abbildung 13.6: Potenzial von KK in den Spielfeldern Sprache, Musik, visuelle Medien und Produktgestaltung

bislang im Fadenkreuz der Automatisierung standen. Wie von Florida ausgeführt, bringen die Kreativen Innovationen im Allgemeinen sehr wirksam voran. Während Arbeit in Produktion und Sachbearbeitung anhand einer Lasterfüllung bemessen wird, schafft kreative Arbeit Neuheit, die günstig für Innovationen ist. In Bezug auf Technik sind Kreative häufig nicht allein frühe Nutzer, vielmehr sind sie auch MitEntwickler, weil sie aufgrund ihrer Neugier und Technologieaffinität in neue Anwendungsgebiete vorstoßen. Sie sind daran beteiligt zu definieren, was überhaupt als „kreativ" gilt. KK können sie folglich als Werkzeug einsetzen, um schneller und professioneller kreative Ergebnisse zu erzielen, auch ein gesteigertes Spektrum an Ausdrucksformen kann daraus resultieren.

Die Beispiele zeigen, dass KK zu einem weiteren Innovationsfeld für die etablierten Player im KI-Bereich wird. IBM, Google und Co. haben die möglichen Potenziale von KK erkannt und versuchen für ihre entsprechenden Schlüsseltechnologien, wie z. B. "Watson", Anwendungen zu finden. Der Einsatz dieser Technologien ermöglicht zweierlei: Sie hilft dabei, die menschliche Wahrnehmung besser zu verstehen sowie 
neue Erkenntnisse durch KI für die Gestaltung von Produkten und neuen Geschäftsmodellen zu verwerten.

Bei der Vielzahl kreativer Tätigkeitsbereiche ist davon auszugehen, dass sich KK unterschiedlich intensiv auf Arbeitsroutinen auswirken wird. Gemessen am wirtschaftlichen Interesse der großen und entwicklungstreibenden Technologie-Konzerne ist davon auszugehen, dass KK zunächst dort zum Zuge kommt, wo die kommerziellen Verwertungschancen des kreativen Outputs am größten sind.

Es ist daher zu erwarten, dass sich die kreative Arbeit in den skizzierten Spielfeldern jeweils unterschiedlich verändern wird:

„Sprache kann mit KK durchmischt werden, neue Bedeutungen werden jedoch vom Menschen geschaffen."

Betrachtet man die Beispiele im Spielfeld "Sprache" durch das analytische Raster aus Abbildung 3.4.1, sind diese vornehmlich in der unteren, linken Ecke zu verorten. Für "WHIM", "MetaphorlsMyBusiness", „PoeTryMe" oder "Sunspring" muss der Mensch jeweils einen relativ präzisen Möglichkeitsraum definieren, damit kreative Ergebnisse produziert werden können. Deutlich wird, dass mittels KI Phrasen, Worte und Buchstaben nach bestimmten Strukturen und Mustern neu angeordnet werden können. Dabei können zwar Kombinationen entstehen, die sich in der gleichen Form noch niemand ausgedacht hat und die originell sind, eine kreative Qualität, die auch in einem wirtschaftlichen Produktionsprozess relevant ist, entsteht hier jedoch nicht bzw. erst, wie im Beispiel von "Sunspring", unter gestaltender Leitung des Menschen.

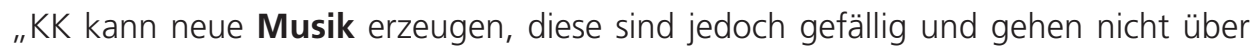
bekannte Formen hinaus."

Musik unterscheidet sich wesentlich von Sprache, weil ihr Sinn nicht über eine begriffliche Bedeutung vermittelt wird, sondern durch eine ästhetische Erfahrung. Damit kann Musik neu angeordnet werden, und es entsteht vergleichsweise einfach ein sinnvoller Zusammenhang, weil etwas gut klingt. Damit hat KK das Potenzial, Neuerungen zu schaffen, die Menschen als sinnvoll bewerten. Wenn also Menschen einen Wirkungsraum festlegen, kann die KI eigenständig kreieren und dabei noch nicht Bekanntes hervorbringen. Die bisher geläufigen Ergebnisse eignen sich z. B. als Untermalung von Werbung oder für Einkaufserlebnisse in Shopping-Centern. Hier sind sie vor allem auch dann interessant, wenn entsprechende GEMA-Gebühren vermieden werden können.

„Mit KK können visuelle Medien schneller gut aussehen, ein neues ,Genie' ist die KK jedoch nicht." 
Die Beispiele im Spielfeld "visuelle Medien" zeigen, dass KK kreative Arbeit deutlich verändern kann. Zum Teil verändert sich die Rolle des Menschen dabei: im Produktionsprozess kann der Mensch vom Schaffenden zum Bewerter wie im Beispiel "Next Rembrandt" oder zum Verwerter wie beim Beispiel "AIR" werden. Kurzfristiger und zunächst gravierender ist jedoch die Geschwindigkeit, mit der etwas Neues hervorgebracht werden kann. Dass ein Musikvideo binnen Stunden neu generiert werden und dabei einen Überraschungseffekt beim Betrachter erzeugen kann, ist möglicherweise nicht im künstlerischen Sinne, wohl aber im Hinblick auf die werbende Wirkung von Musikvideos und die wirtschaftliche Struktur ihres Produktionsprozesses von Bedeutung. Ähnliches gilt für die Kl-gestützte Anwendung von visuellen Filtern, die Bilder in kürzester Zeit bearbeiten inkl. eines, unter Umständen kurzweiligen, Neuheitseffekts. In diesem Spielfeld steht die Gruppe der Kreativen somit vor einer Aufgabe, die sie gewöhnt ist: sich neue Technologien anzueignen und diese schnell innerhalb eines gegeben Produktionsprozesses anzuwenden.

"Die kreative Verarbeitung von Werkstoffen in der Produktgestaltung wird durch KK grundsätzlich erneuert: Algorithmen bewältigen das Spannungsfeld zwischen materiellen Anforderungen und Ästhetik; sie führen den Menschen zu neuen Designprinzipien."

In der Produktgestaltung entfaltet KK die bislang stärkste Wirkung und lässt ihre Potenz aufscheinen, systemische Neuerungen zu schaffen. In den Beispielen liegt der schöpferische Akt des Designs beim Algorithmus, während der Mensch lediglich den Möglichkeitsraum absteckt und Informationen anordnet. Diesen Raum „erkunden" die Algorithmen. Daraus gehen Entwürfe hervor, die sich Menschen allein so nicht hätten ausdenken könnten. Insbesondere das Beispiel des Autochassis zeigt, dass die Güte der Entwürfe mit der Qualität der Daten ansteigt. Wenn ein Computer mehr Input erhält, entstehen für ihn auch mehr Möglichkeiten. Auf Grundlage dieser Bedingungen können Algorithmen Entwürfe entwickeln, deren Neuheit weit über die Gestaltung von Oberflächen hinausgeht. Der Mensch wird hier zum Zuarbeiter und ordnet Informationen an, die dann den Rahmen bilden, in dem die Technik systemische Neuerungen und gänzlich neue Lösungswege sowie ästhetische Prinzipien schafft. Das Spannungsfeld zwischen materiellen Anforderungen und Ästhetik, das in diesem Spielfeld besonders ausgeprägt ist, begünstigt die Eingriffstiefe der KK.

\section{Bedingungen für Künstliche Kreativität}

Alle Spielfelder entwickeln sich nicht losgelöst von allgemeinen gesellschaftlichen Trends sowie materiellen und kulturellen Bedingungen. Dabei lassen sich einige Punkte, die die Durchsetzung von KK unterstützen, zusammenfassen: 
Die Bedeutung von kreativer Arbeit wächst in allen Feldern mit dem gesellschaftlichen Trend zur Individualisierung, der die Orientierung an neuen, besonderen und exklusiven Produkten nährt.

Die technischen Voraussetzungen zur Implementierung von KI sind bereits dann aussichtsreich, wenn kreative Arbeit stark technisch geprägt ist. Künstliche Kreativität öffnet hier die Möglichkeit, kreative Kompetenzen stärker zugunsten des Computers aufzuteilen.

Über Qualität wird die Kreativbranche intensiver diskutieren: Als Bewertungsraster für die Sinnhaftigkeit des Ergebnisses zieht KK Wahrscheinlichkeiten heran. Offen ist, inwiefern man langfristig einen computerbasierten Output als kreativ bezeichnen kann oder eher ein Massengeschmack angesprochen wird.

Der Konkurrenzdruck unter Kreativdienstleistern ist hoch, sodass Unternehmen nach neuen Wettbewerbsvorteilen suchen. Für die Produktionsprozesse kreativer Arbeit bedeutet dies hohe Anforderungen an Geschwindigkeit, bei gleichzeitig hohen Ansprüchen an Design und technischer Funktionalität.

Die Branchen bestehen aus vielen kleinen und mittleren Unternehmen, deren Arbeitsprozesse flexibel sind und die neue Technologien schnell aufgreifen und implementieren können.

Die Kreativarbeit ist aufgrund ihrer Kultur eine Spielwiese, auf der neue Sachen ausprobiert werden können. So lassen sich neue Konstellationen assistierender und/oder leitender Zusammenarbeit von Menschen und Algorithmen prototypisch erproben und in andere Tätigkeitsbereiche übertragen. 


\section{Literatur}

Boden, Margaret A. (1992): Die Flügel des Geistes. Kreativität und künstliche Intelligenz. München: Artemis und Winkler.

Boden, Margaret A. (1998): Creativity and artificial intelligence. In: Artificial Intelligence 103 (1-2), S. 347-356. DOI: 10.1016/S0004-3702(98)00055-1.

Florida, Richard L. (2003): The Rise of the Creative Class. And How It's Transforming Work, Leisure, Community, and Everyday Life: Brilliance Corp.

Gervas (2013): Online verfügbar unter http://nil.fdi.ucm.es/sites/default/files/GervasAISB2013CRC.pdf, zuletzt geprüft am 22.06.2018.

Joas, Hans (1996): Die Kreativität des Handelns. Frankfurt am Main: Suhrkamp.

Lévi-Strauss (1973): Das wilde Denken. Frankfurt am Main: Suhrkamp.

Reckwitz, Andreas (2014): Die Erfindung der Kreativität. Zum Prozess gesellschaftlicher Ästhetisierung. 4. Aufl. Berlin: Suhrkamp (Suhrkamp-Taschenbuch Wissenschaft).

Söndermann, Michael; Backes, Christoph; Arndt, Olaf; Brünink, Daniel (2009): Kultur- und Kreativwirtschaft: Ermittlung der gemeinsamen charakteristischen Definitionselemente der heterogenen Teilbereiche der „Kulturwirtschaft" zur Bestimmung ihrer Perspektiven aus volkswirtschaftlicher Sicht. Hg. v. Bundesministerium für Wirtschaft und Technologie. Köln, Bremen, Berlin.

Stubbe, Julian (2017): Articulating Novelty in Science and Art. The Comparative Technography of a Robotic Hand and a Media Art Installation. Wiesbaden: Springer VS.

Wiggins, Geraint A. (2006): Searching for computational creativity. In: New Gener Comput 24 (3), S. 209-222. DOI: 10.1007/BF03037332.

\section{(c) (i)}

Dieses Kapitel wird unter der Creative Commons Namensnennung 4.0 International Lizenz http://creativecommons.org/licenses/by/4.0/deed.de) veröffentlicht, welche die Nutzung, Vervielfältigung, Bearbeitung, Verbreitung und Wiedergabe in jeglichem Medium und Format erlaubt, sofern Sie den/die ursprünglichen Autor(en) und die Quelle ordnungsgemäß nennen, einen Link zur Creative Commons Lizenz beifügen und angeben, ob Änderungen vorgenommen wurden.

Die in diesem Kapitel enthaltenen Bilder und sonstiges Drittmaterial unterliegen ebenfalls der genannten Creative Commons Lizenz, sofern sich aus der Abbildungslegende nichts anderes ergibt. Sofern das betreffende Material nicht unter der genannten Creative Commons Lizenz steht und die betreffende Handlung nicht nach gesetzlichen Vorschriften erlaubt ist, ist für die oben aufgeführten Weiterverwendungen des Materials die Einwilligung des jeweiligen Rechteinhabers einzuholen. 